\title{
Vaccine development for rheumatoid arthritis
}

\author{
Charles J. Malemud* \\ Department of Medicine, Division of Rheumatic Diseases, Case Western Reserve University School of Medicine, USA
}

\begin{abstract}
Rheumatoid arthritis (RA) is an autoimmune disorder characterized by chronic synovial joint inflammation, destruction of articular cartilage and erosion of subchondral bone. Major advances have been made in the medical management of RA with the introduction of various types of biologic drugs which neutralize the activity of pro-inflammatory cytokines as well as T-cell and B-cell proliferation. Furthermore, a Janus Kinase small molecule inhibitor which suppresses Signal Transducers and Activators of Transcription protein phosphorylation has now been added to the RA drug armamentarium. Despite the development of these novel drugs, there are still no "preventive" measures available for RA. The recent discovery that autoantibodies are produced against cyclic citrullinated proteins/peptides in a significant number of individuals with or without undifferentiated arthritis which can precede the clinical symptoms of RA has provided an impetus for a prophylactic vaccine therapy for RA. Experimental evidence which showed that various vaccine formulations ameliorated arthritis in well-validated animal models has now led to the development of "Rheumavax" which has already been evaluated with promising results in a phase I RA clinical trial.
\end{abstract}

\section{Introduction}

Rheumatoid arthritis (RA) is a systemic, chronically progressive autoimmune disorder characterized by defective innate and adaptive immune responses [1-5]. Synovial joint inflammation, destruction of subchondral bone and articular cartilage, the latter occurring via degradation of cartilage extracellular matrix (ECM) as well as altered soft tissue structures, including those required to maintain the function of ligaments, capsule and tendons are also hallmarks of RA pathology [6-8].

In RA the synovial joint structural damage is also accompanied by "apoptosis-resistance" in the hyperplastic synovial tissue [9-12] as well as by an increase in the number of apoptotic articular chondrocytes, which significantly affects attempts at cartilage repair [13-15]. From a molecular and pathophysiologic perspective the destruction of the RA synovial joints is further facilitated by aberrant signal transduction [16], by up-regulation of pro-inflammatory cytokine gene expression [17], and by increased chemokine and adhesion protein synthesis $[18,19]$. The increase in pro-inflammatory cytokines, exemplified by tumor necrosis factor- $\alpha$ (TNF- $\alpha$ ), interleukin- $1 \beta$ (IL-1 $\beta$ ) and IL-6 gene expression causes elevated matrix metalloproteinase (MMP) synthesis to occur which, in RA, is typically responsible for ECM protein degradation in articular cartilage $[18,20]$. However, defects in receptor activator nuclear factor- $\mathrm{kB}$ ligand/receptor activator of nuclear factor- $\kappa B$ (RANKL/RANK), and the decoy receptor of RANKL, osteoprotegerin, signaling brought about by the action of pro-inflammatory cytokines is the primary mechanism responsible for subchondral bone erosions [21].

Therefore, based on these considerations it could have been anticipated that therapeutic neutralizing monoclonal antibodies and/ or fusion proteins would be developed to block TNF- $\alpha$, IL- $1 \beta$ and IL6-mediated signaling for the medical treatment of RA [22]. Indeed, biologic drugs are now routinely employed in clinical practice in the treatment of RA. These biologics are used in addition to the more conventional first-line therapies for RA which includes methotrexate, several types of anti-malarial drugs, prednisone, non-steroidal anti- inflammatory drugs and sulphasalazine [23]. Additional RA treatment modalities also now include using these biologic drugs in combination therapies. Several of these drugs are targeted therapies. They include leflunomide, a general pyrimidine synthesis inhibitor [24] designed to inhibit immune cell proliferation or drugs targeted to inhibit T-cell proliferation with the T-cell activation modulator, abatacept [25] or B-cells using the Blys modulator, atacicept [26], B-cell proliferation using the anti-CD20 monoclonal antibody, rituximab [27] or tocilizumab, a monoclonal antibody that neutralizes the action of IL-6 [28].

More recently, tofacitinib, the first small molecule inhibitor to be employed in the therapy of RA, was developed to selectively target Janus Kinases [29-31]. This drug development strategy was primarily founded on the results of basic research which showed that the interaction between IL- 6 and the IL- 6 receptor, the latter found either in a complex with glycoprotein-130 (i.e. the IL-6/IL-6R/gp130 complex) or with membrane-bound IL-6R or soluble IL-6R activated the Janus Kinase/Signal Transducers and Activators of Transcription (JAK/STAT) pathway [29], and in doing so caused an increase in proinflammatory cytokine gene expression [31].

Although these multitudes of clinically efficacious RA therapies are now routinely used and have revolutionized the medical therapy of RA, there have been several additional recent advances which point the way to an entirely innovative strategy for "preventing" RA from developing in the first place. Thus, recent evidence has shown that cyclic citrullinated proteins (CCP) were likely to be the arthritogenic proteins which are the critical protein component for developing RA

Correspondence to: Charles J. Malemud, Professor Medicine and Anatomy, Case Western Reserve University, USA, Tel: 0012165361945 ; E-mail: cjm4@case.edu

Key words: autoimmune, arthritis, cytokines, tolerance, vaccine

Received: November 12, 2015; Accepted: December 07, 2015; Published: December 11, 2015 
[32]. In that regard, most notably, autoantibodies directed against CCP protein/peptides (ACPA) were found in the sera of about $70 \%$ of RA patients as well as in the sera of individuals who eventually developed RA. In many of these cases ACPAs were found in these sera several years prior to the appearance of RA clinical symptoms [32]. These novel discoveries resulted in a new venture suggesting that prophylactic vaccination might be the long sought after "cure" for RA.

\section{The ACPA connection}

Citrulline is an $\alpha$-amino acid and a key intermediate in the urea cycle. However, several proteins contain citrulline resulting from a post-translational modification whereby citrulline is produced from arginine via the action of peptidylarginine deiminase [33].

ACPAs are produced against various autoantigens, including, fibrinogen, vimentin, Type II collagen, and $\alpha$-enolase [34] as well as against filaggrin, fibronectin and histones. Recent evidence also showed that ACPAs were representative in a significant number of RA patients and importantly, ACPA-positive individuals with early undifferentiated arthritis may have a higher risk for developing RA [35]. However, as pointed out by Coenen et al. [34] it will also be necessary to competently evaluate the clinical presentation of patients who are ACPA-positive since patients presenting with psoriatic arthritis may also be ACPA-positive.

ACPA production was also found to be associated with the HLADRB1 shared epitope and PTPN22 1858T allele [35] both of which constitute known RA susceptibility loci. Of note, Uçar et al. [36] showed in a population of Turkish patients that differences in the pattern of HLA-DRB1 alleles was, indeed, dependent on the presence or absence of ACPAs. HLA-DRB $1^{\star} 04$ and HLA-DRB1 ${ }^{\star} 09$ were associated with ACPA-positive individuals as well as ACPA-positive RA patients, whereas $\mathrm{DRB} 1^{\star} 01$ and $\mathrm{DRB} 1{ }^{\star} 04$ allele levels were higher in rheumatoid factor-positive, ACPA-negative RA patients. Furthermore, the results showed that associated and protective HLA-DRB1 allele distributions were in line with differing ACPA/rheumatoid factor patterns. In that regard, the HLA-DRB1* 13 allele alone was found to be protective against production of anti-CCP autoantibodies and rheumatoid factor. Thus, when this data is considered together, they may account for the critical finding that the HLA-DRB1 shared epitope underlies the capacity of T-cells to recognize citrullinated proteins [36].

Moreover, ACPAs are also pathologic. The evidence for this appears to be substantial and compelling since ACPAs were demonstrated to induce several critical components of RA pathology. These included the production of TNF- $\alpha$ by activated macrophages, enhanced differentiation of osteoclasts, IL-8, a pro-inflammatory biomarker of inflammation, as well as having the capacity to induce complement activation [32]. All of these factors promote synovial joint progression of disease typical of RA.

A significant advance in our understanding of how prophylactic vaccination could ultimately result in the future treatment of RA recently arose when Benham et al. [37] conducted a phase I clinical safety and efficacy trial in Australia. These investigators studied a vaccine construct comprised of autologous immunomodulatory dendritic cells (DCs) that had been modified with an NF- $\kappa B$ inhibitor and then exposed to 4 citrullinated peptide antigens. This immunotherapeutic preparation designated "Rheumavax" was administered by intradermal injection to 18 individuals in a single-center open-label phase I clinical trial. These subjects had previously been shown to be leukocyte antigen genotype-positive with citrullinated peptide-specific autoimmunity.
The major result of this study was that "Rheumavax" was well-tolerated and had few adverse events. From an immunologic perspective, the number of effector T-cells was reduced by "Rheumavax" which was accompanied by an increased ratio of T-regulatory $\left(\mathrm{T}_{\text {reg }}\right)$ cells to effector T-cells 1 month following vaccination. Moreover, "Rheumavax" reduced the levels of IL-15, IL-29, CX3CL1 and CXCL11 in serum whilst also reducing IL-6 responses to vimentin (447-455)-Cit450 by T-cells compared to a control group. Importantly, "Rheumavax" did not exacerbate RA flares. On the contrary, "Rheumavax" decreased the DAS28 score in the RA group within 1 month. Thus, the results of this phase I clinical trial should provide the impetus for a further analysis of this ACPA-autoantigen-based vaccine as a potential immunomodulatory therapy for RA.

\section{Alternative "Vaccine" Strategies for RA}

\section{Anti-cytokine and Anti-autoreactive T-cell vaccines}

The development of anti-cytokine vaccines employs a strategy whereby a cytokine is targeted which has been shown to be pathogenic and over-expressed in RA and other autoimmune disorders [38]. Thus, among the first vaccine designs in this group was one targeted against the pro-inflammatory macrophage inhibitory protein (MIF) as well as the IL-2 receptor subunit [39]. Necessarily, the development of these anti-cytokine vaccines will not proceed forward unless they are first rigorously tested in well-validated animal models of human RA. Thus, it is useful to consider that results from these animal model studies must be interpreted with caution since the initiating autoantigen employed to produce the arthritic response in these animal models, as well as the profile of $\mathrm{T}_{h} 1, \mathrm{~T}_{h} 2$, and $\mathrm{T}_{h} 17$ cells after vaccination may be quite dissimilar to the $\mathrm{T}$-cell repertoire found in human inflammatory arthritis [40].

IL-23 is a pro-inflammatory cytokine required for the development of $\mathrm{T}_{h} 17$ cells [41]. IL-23 shares a subunit with IL-12 known as IL$12 / 23$ p40 and comprises a specific subunit of IL-23p19. Ratsimandresy et al. [42] used bioinformatics modeling of the murine IL-23p19 subunit and 2 peptides in the receptor interacting domain coupled to keyhole limpet hemocyanin to create 2 vaccines, IL23-K1 and IL23-K2. Mice immunized with IL23-K1 produced greater amounts of anti-IL23 antibodies than mice immunized with IL23-K2. DBA/1 mice with collagen-induced arthritis (CIA) were then vaccinated. The results of this study indicated that mice immunized with IL23-K1 produced more splenic IL-10 than with IL23-K2 however, without altering IL-17. Furthermore, IL23-K1 was highly protective against joint destruction and inflammation, although the population of T-cells in the spleen were not modulated by the IL23-K1 vaccine. These results suggested that a specific IL23p19 vaccine may be useful for future human RA immunotherapy studies, although no follow-up studies of IL23-K1 have appeared in the PubMed database by 2015 .

Yuan et al. [43] generated a human RANKL-TNF-like core fusion protein, termed the RTFP-2 vaccine. RTFP-2-generated antisera when added to L929 cells decreased TNF- $\alpha$-induced apoptosis as well as producing a complete inhibition of osteoclastogenesis in vitro. Of note, the RTFP-2 vaccine also significantly reduced the severity of mouse CIA through its capacity to inhibit inflammation and bone resorption. However, no additional publications regarding the RTFP-2 vaccine was listed in the PubMed database since these results were first published in 2012 .

Another innovative vaccine strategy would employ autoreactive $\mathrm{T}$-cells to induce self-tolerance [44]. In that regard, this vaccine design 
would rely on confirming that arthritogenic T-cells, which have evaded negative selection during $\mathrm{T}$-cell development, would now recognize arthritogenic antigens. Increased levels of B-cell activating factor (BAFF) and a proliferation inducing ligand (APRIL) are associated with autoimmune disorders such as systemic lupus erythematosus and RA [45]. Thus, BAFF and APRIL have been linked to B-cell survival, differentiation, proliferation and antibody production which are likely to influence RA autoimmunity. To approach this component of RA pathogenesis, a BAFF adjuvant-free autovaccine was developed by coupling a $\mathrm{T}$ helper cell epitope, termed PADRE to the $\mathrm{N}$-terminus domain of the BAFF extracellular domain (PADRE-BAFF) with the production of this fusion protein in E. coli [46]. The PADRE-BAFF vaccine yielded high titers of neutralizing BAFF antibodies whilst also ameliorating arthritis in rats in which the arthritis was induced with complete Freund's adjuvant.

Finally, another innovative strategy in this vaccine category took advantage of evidence which showed that the endocytic receptor DEC205 on DCs, termed DEC205 ${ }^{+}$DCs may be a suitable vehicle for inducing immune tolerance [47]. Thus, Spiering et al. [48] showed that the targeting of human cartilage proteoglycan peptide 70-84 with DEC $205^{+}$DCs protected mice from proteoglycan-induced arthritis. In that respect, the main effect of DEC205 ${ }^{+}$DCs was to suppress germinal center B-cell support by proteoglycan-specific follicular helper T-cells. Moreover, pretreatment of mice with proteoglycan 70-84 peptide, using this DEC205 targeting approach induced T-cell deletion and/ or anergy. Furthermore, the proportion of $\mathrm{T}_{\text {reg }}$ cells were found to be increased at the same time. Overall, these results suggested that prospective tolerogenic DEC205 ${ }^{+} \mathrm{DC}$ vaccination might become a useful immunotherapeutic modality for RA.

\section{Type II collagen vaccines}

In 2010, Zimmerman et al. [49] published the results of a preliminary study in mouse CIA in which a "Ligand Epitope Antigen Presentation System" (LEAPS) was employed to produce a therapeutic vaccine. LEAPS is a system for producing vaccine constructs involving a peptide hetero-conjugate. Thus, this vaccine designated "CEL-2000" was constructed from a peptide of Type II collagen containing amino acids, 254-273, which was then attached to an immune or T-cell binding protein from human $\beta 2$ microglobulin. The results from study in mouse CIA indicated that "CEL-2000" limited the progression of arthritis as evidenced by a reduction in the 'arthritis-index-score' as well as by microscopic examination of the inflamed synovial joints. Of note, "CEL-2000" also modulated selective serum cytokine levels. In that regard, "CEL-2000"-treated mice with CIA showed evidence of increased levels of IL-12p70 and IL-10, both of which could be considered to be an anti-inflammatory response. Interestingly, this result was not obtained when mice with CIA were administered etanercept, an anti-TNF- $\alpha$ fusion protein employed in the therapy of human RA. However, the PubMed database contained no additional published results of any follow-up studies of "CEL-2000" either in arthritis animal models or in human safety clinical trials.

A therapeutic DNA vaccine encoding chicken Type II collagen (CCII), termed pcDNA-CCOL2A1 was compared with methotrexate to evaluate their effects on cellular and humoral immune responses in normal rats [50]. In this study, vaccinating normal rats with pcDNACCOL2A1 did not result in the production of anti-CCII antibodies. Furthermore, no significant changes in the levels of several proinflammatory cytokines or the chemotactic protein, MCP-1 or other proteins implicated in RA, including, MIP-1 1 , Regulated on Activation in Normal T-cell Expressed (RANTES), RANKL, IL-4 or IL-10 were noted. In contrast, transforming growth factor- $\beta$ levels were increased whereas interferon $-\gamma$ and TNF- $\alpha$ levels were decreased. At the cellular level, pcDNA-CCOL2A1 did not alter the percentages of $T_{h} 1, T_{h} 2$ or $\mathrm{T}_{h} 17$ cells. However, pcDNA-CCOL2A1 did reduce the number of $\mathrm{T}_{\text {res }}$ cells whilst also increasing CD4 ${ }^{+} \mathrm{T}$-cells. Thus, the pcDNA-CCOL2A1 vaccine did not appear to markedly alter parameters of $\mathrm{T}$-cell responses in normal rats indicating that future studies in rat arthritis models may be warranted.

\section{Conclusions and future perspectives}

A variety of vaccine development strategies have been employed wherein their efficacy in animal models of experimentally-induced inflammatory arthritis have been tested. In particular, one vaccine in currently in development, called, "Rheumavax" has actually completed a phase I safety trial with promising results. However, a note of caution is also appropriate here. In that regard, the results of several studies reviewed here $[42,43,49]$ are, in some cases, 5 years old [49] with no evidence from the PubMed database of follow-up results since that time. This literature search suggested either those additional studies were performed but the results did not confirm preliminary data or that further development of these vaccine constructs were not pursued for other reasons. Therefore, it is difficult to predict which if any future studies will rely on the same or different vaccine development paradigms for immunotherapeutic management of RA. We must also be cognizant of the possible effects of immunotherapeutic vaccines for RA on the clinical efficacy of other vaccination protocols designed to protect RA patients against infection. However, future studies of immunotherapeutic vaccines in development may provide a promising clinical scenario for eventually incorporating a prophylactic therapy into the overall management of undifferentiated arthritis and RA.

\section{References}

1. Firestein GS (2005) Immunologic mechanisms in the pathogenesis of rheumatoid arthritis. J Clin Rheumatol 11: S39-S44. [Crossref]

2. Toh ML, Miossec P (2007) The role of T cells in rheumatoid arthritis: new subsets and new targets. Curr Opin Rheumatol 19: 284-288. [Crossref]

3. Bugatti S, Codullo V, Caporali R, Montecucco C (2007) B cells in rheumatoid arthritis Autoimmun Rev 6: 482-487. [Crossref]

4. Szekanecz Z, Koch AE (2007) Macrophages and their products in rheumatoid arthritis Curr Opin Rheumatol 19: 289-295. [Crossref]

5. Malemud CJ (2011) Molecular mechanisms in rheumatic diseases. Rationale for nove drug development - Introduction. Anti-Inflamm Anti-Allergy Agents Med Chem 10: 73 77. [Crossref]

6. Karouzakis E, Neidhart M, Gay RE, Gay S (2006) Molecular and cellular basis of rheumatoid joint destruction. Immunol Lett 106: 8-13. [Crossref]

7. Huber LC, Distler O, Tarner I, Gay RE, Gay S, et al. (2006) Synovial fibroblasts: key players in rheumatoid arthritis. Rheumatology (Oxford) 45: 669-675. [Crossref]

8. Fox DA, Gizinski A, Morgan R, Lundy SK (2010) Cell-cell interactions in rheumatoid arthritis synovium. Rheum Dis Clin North Am 36: 311-323. [Crossref]

9. Liu H, Pope RM (2004) Apoptosis in rheumatoid arthritis: friend or foe. Rheum Dis Clin North Am 30: 603-625, x. [Crossref]

10. Malemud CJ, Gillespie HJ (2005) The role of apoptosis in arthritis. Curr Rheum Rev 131-142.

11. Korb A, Pavenstädt H, Pap T (2009) Cell death in rheumatoid arthritis. Apoptosis 14 447-454. [Crossref]

12. Malemud CJ (2011) Apoptosis resistance in rheumatoid arthritis synovial tissue. J Clin Cell Immunol S3:006.

13. Malemud CJ, Schulte ME (2008) Is there a final common pathway for arthritis? Future Rheumatol 3: 253-268. 
14. Hutcheson J, Perlman H (2008) Apoptosis regulators and RA. Curr Rheum Rev 4: 254 258.

15. Lewis AC, Malemud CJ (2011) Correction of defective apoptosis in arthritis by pharmacologic strategies. Focus on altering the activity of inhibitor of apoptosis protein. In: Pandalai SG (Ed.) Recent Research Developments in Pharmacology, 69-84

16. Wylie MA, Malemud CJ (2013) Perspective: Deregulation of apoptosis in arthritis by altered signal transduction. Int J Clin Rheumatol 8: 483-490.

17. Malemud CJ (2013) Regulation of chondrocyte matrix metalloproteinase gene expression. In: Role of Proteases in Cellular Dysfunction. Dhalla NS, Chakraborti S (Eds.), Springer Science, UK, 63-77.

18. Malemud CJ, Reddy SK (2008) Targeting cytokines, chemokines and adhesion molecules in rheumatoid arthritis. Curr Rheum Rev 4: 219-234.

19. Malemud CJ (2008) Defining novel targets for intervention in rheumatoid arthritis: An overview. Curr Rheum Rev 4: 214-218.

20. Malemud CJ (2012) Targeted drug development for arthritis. Future Med Chem 4: 701-703. [Crossref]

21. Leibbrandt A, Penninger JM (2008) RANK/RANKL: regulators of immune responses and bone physiology. Ann N Y Acad Sci 1143: 123-150. [Crossref]

22. Gibbons LJ, Hyrich KL (2009) Biologic therapy for rheumatoid arthritis: clinical efficacy and predictors of response. BioDrugs 23: 111-124. [Crossref]

23. Feely MG, Erickson A, O’Dell JR (2009) Therapeutic options for rheumatoid arthritis Expert Opin Pharmacother 10: 2095-2106. [Crossref]

24. Kumar P, Banik S (2013) Pharmacotherapy options in rheumatoid arthritis. Clin Med Insights Arthritis Musculoskelet Disord 6: 35-43. [Crossref]

25. Ostör AJ (2008) Abatacept: a T-cell co-stimulation modulator for the treatment of rheumatoid arthritis. Clin Rheumatol 27: 1343-1353. [Crossref]

26. Richez C, Truchetet ME, Schaeverbeke T, Bannwarth B (2014) Atacicept as an investigated therapy for rheumatoid arthritis. Expert Opin Investig Drugs 23: 12851294. [Crossref]

27. Caporali R, Caprioli M, Bobbio-Pallavicini F, Bugatti S, Montecucco C (2009) Long term treatment of rheumatoid arthritis with rituximab. Autoimmun Rev 8: 591-594. [Crossref]

28. Tanaka T, Kishimoto T (2013) Immunotherapy of tocilizumab for rheumatoid arthritis. J Clin Cell Immunol S6: 001.

29. Malemud CJ, Pearlman E (2009) Targeting JAK/STAT signaling pathway in inflammatory diseases. Curr Signal Transduct Ther 4: 201-221.

30. Malemud CJ, Blumenthal DE (2014) Protein kinase small molecule inhibitors for rheumatoid arthritis: Medicinal chemistry/Clinical perspectives. World J Orthop 5: 496-503. [Crossref]

31. Malemud CJ (2015) The small molecular weight inhibitor of protein kinase revolution for the treatment of rheumatoid arthritis. In: Drug Discovery and Development- From Molecules to Medicine. Vallisuta O, Olimat S (Eds.), InTech Publishing, Rijeka, CROATIA, 163-179

32. Sakkas LI, Bogdanos DP, Katsiari C, Platsoucas CD4 (2014) Anti-citrullinated peptides as autoantigens in rheumatoid arthritis-relevance to treatment. Autoimmun Rev 13: 1114-1120. [Crossref]

33. Wegner N, Lundberg K, Kinloch A, Fisher B, Malmström V, et al. (2010) Autoimmunity to specific citrullinated proteins gives the first clues to the etiology of rheumatoid arthritis. Immunol Rev 233: 34-54. [Crossref]
34. Coenen D, Verschueren P, Westhovens R, Bossuyt X (2007) Technical and diagnostic performance of 6 assays for the measurement of citrullinated protein/peptide antibodies in the diagnosis of rheumatoid arthritis. Clin Chem 53: 498-504. [Crossref]

35. Szodoray P, Szabó Z, Kapitány A, Gyetvai A, Lakos G, et al. (2010) Anti-citrullinated protein/peptide autoantibodies in association with genetic and environmental factors as indicators of disease outcome in rheumatoid arthritis. Autoimmun Rev 9: 140-143. [Crossref]

36. Uçar F, Çapkin E, Karkucak M, Yücel B, Sönmez M, et al. (2012) Associations of HLADRB1 alleles with anti-citrullinated protein antibody-positive and anti-citrullinated protein antibody-negative rheumatoid arthritis in northern east part of Turkey. Int $J$ Rheum Dis 15: 538-545. [Crossref]

37. Benham H, Nel HJ, Law SC, Mehdi AM, Street S, et al. (2015) Citrullinated peptide dendritic cell immunotherapy in HLA risk genotype-positive rheumatoid arthritis patients. Sci Transl Med 7: 290ra87. [Crossref]

38. Delavallée L, Duvallet E, Semerano L, Assier E, Boissier MC (2010) Anti-cytokine vaccination in autoimmune diseases. Swiss Med Wkly 140: w13108. [Crossref]

39. Shabahang S, Li AF, Escher A (2010) Recent patents on immunoregulatory DNA vaccines for autoimmune diseases and allograft rejection. Recent Pat DNA Gene Seq 4: 122-131. [Crossref]

40. Rosenthal KS, Mikecz K, Steiner HL 3rd, Glant TT, Finnegan A, et al. (2015) Rheumatoid arthritis vaccine therapies: perspectives and lessons from therapeutic ligand epitope antigen presentation system vaccines for models of rheumatoid arthritis Expert Rev Vaccines 14: 891-908. [Crossref]

41. Fragoulis GE, Siebert S, McInnes IB (2015) Therapeutic Targeting of IL-17 and IL-23 Cytokines in Immune-Mediated Diseases. Annu Rev Med . [Crossref]

42. Ratsimandresy RA, Duvallet E, Assier E, Semerano L, Delavallée L, et al. (2011) Active immunization against IL-23p19 improves experimental arthritis. Vaccine 29: 9329-9336. [Crossref]

43. Yuan H, Qian H, Liu S, Zhang X, Li S, et al. (2012) Therapeutic role of a vaccine targeting RANKL and TNF- $\alpha$ on collagen-induced arthritis. Biomaterials 33: 81778185. [Crossref]

44. Wang D, Li Y, Liu Y, Shi G (2015) The role of autoreactive T cell in the pathogenesis of rheumatoid arthritis and implications for $\mathrm{T}$ cell targeted vaccine therapy. Minerva Med 106: 157-167. [Crossref]

45. Morais SA, Vilas-Boas A, Isenberg DA (2015) B-cell survival factors in autoimmune rheumatic disorders. Ther Adv Musculoskelet Dis 7: 122-151. [Crossref]

46. Feng GD, Xue XC, Gao ML, Wang XF, Shu Z, et al. (2014) Therapeutic effects of PADRE-BAFF autovaccine on rat adjuvant arthritis. Biomed Res Int 2014: 854954. [Crossref]

47. Sehgal K, Dhodapkar KM, Dhodapkar MV (2014) Targeting human dendritic cells in situ to improve vaccines. Immunol Lett 162: 59-67. [Crossref]

48. Spiering R, Margry B, Keijzer C, Petzold C, Hoek A, et al. (2015) DEC205+ Dendritic Cell-Targeted Tolerogenic Vaccination Promotes Immune Tolerance in Experimental Autoimmune Arthritis. J Immunol 194: 4804-4813. [Crossref]

49. Zimmerman DH, Taylor P, Bendele A, Carambula R, Duzant Y, et al. (2010) CEL2000: A therapeutic vaccine for rheumatoid arthritis arrests disease development and alters serum cytokine/chemokine patterns in the bovine collagen type II induced arthritis in the DBA mouse model. Int Immunopharmacol 10: 412-421. [Crossref]

50. Xiao Z, Juan L, Song Y, Zhijian Z, Jing J, et al. (2015) Evaluation of humoral and cellular immune responses to a DNA vaccine encoding chicken type II collagen for rheumatoid arthritis in normal rats. Hum Vaccin Immunother 11: 938-945. [Crossref]

Copyright: (C2015 Malemud CJ. This is an open-access article distributed under the terms of the Creative Commons Attribution License, which permits unrestricted use, distribution, and reproduction in any medium, provided the original author and source are credited. 